\title{
Dietary N-3 Polyunsaturated Fatty Acids Decrease Biliary Cholesterol Saturation in Gallstone Disease
}

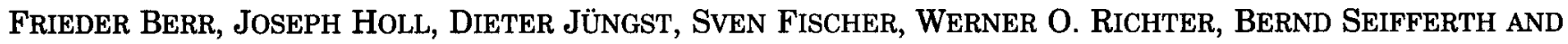 \\ GuSTAV PAUMGARTNER \\ Department of Medicine II, Klinikum Grosshadern, University of Munich, D-8000 Munich 70, Germany
}

\begin{abstract}
Because fatty acid composition of biliary phospholipids influences cholesterol secretion into bile, we investigated whether replacement of n-1 monounsaturated or $n-6$ polyunsaturated fatty acids with n-3 polyunsaturated fatty acids in biliary phosphatidylcholines reduces supersaturation with cholesterol and prevents precipitation of cholesterol crystals in bile of gallstone patients. Seven patients with radiolucent gallstones in functioning gallbladders were studied before (control) and after 5 wk of dietary supplementation with marine fish oil $(11.3 \mathrm{gm} /$ day $=3.75 \mathrm{gm} \mathrm{n}-3$ polyunsaturated fatty acids/day). Duodenal bile was collected for analysis during intravenous infusion of cholecystokinin. Gallbladder emptying in response to cholecystokinin was comparable before and during intake of $n-3$ polyunsaturated fatty acids. Intake of $n-3$ polyunsaturated fatty acids increased $(p<0.001)$ the fractions of eicosapentaenoic and docosahexaenoic acids and decreased the fractions of linoleic $(p<0.001)$ and arachidonic acids $(p<0.02)$ in biliary phospholipids. Concomitantly, the molar ratio of cholesterol to phospholipids decreased $(-19 \% ; p<0.05)$. As a consequence, the cholesterol saturation index was reduced by $-25 \%$ $(p=0.01)$, from $1.60 \pm 0.44$ to $1.24 \pm 0.38$. However, in vitro nucleation time of duodenal bile was not prolonged. The decrease in cholesterol saturation was not sufficient to prevent nucleation of cholesterol crystals in bile of gallstone patients. In conclusion, our data suggest that cholesterol saturation can be influenced by the fatty acid composition of the phosphatidylcholines secreted in bile. (HEPATOLOGY 1992;16:960-967.)
\end{abstract}

Formation of gallstones requires at least two stepssecretion of bile supersaturated with cholesterol and nucleation of cholesterol crystals (1). Saturation of bile with cholesterol can be decreased with intake of bile acids in use for gallstone dissolution; bile acids become enriched in bile and lower the secretory ratio of cholesterol to bile acids $(2,3)$. No attempts have yet been

Received May 30, 1991; accepted June 4, 1992

This work was supported by grant Be 890/2-2 from the Deutsche Forschungsgemeinschaft and by Nycomed Arzneimittel GmbH, Munich, Germany.

Part of this work has been published in abstract form (HEPATOLOGY 1990;12:898).

Address reprint requests to: Dr. Frieder Berr, Medizinische Klinik II, Klinikum Grosshadern, Marchioninistrasse 15, D-8000 Munich 70, Germany. 31/1/40082 made in cholesterol gallstone disease to lower cholesterol saturation of bile by modification of biliary phospholipids, even though the secretion of cholesterol in bile is linked to that of phospholipids (1, 3-6).

Approximately $95 \%$ of biliary phospholipids are phosphatidylcholines, which contain an unsaturated fatty acid in position sn-2 and, almost without exception, a saturated fatty acid in position sn-1 (7). The constituents in position sn-2 are quite varied and, for the most part, are polyunsaturated fatty acids (PUFAs) (7). They can be influenced by the type of PUFA in the diet (8-13) because the two families-n-3 PUFAs and n-6 PUFAs - are essential fatty acids that cannot be interconverted in mammals (14). Dietary n-3 PUFAs were incorporated into biliary phosphatidylcholines; they lowered cholesterol saturation of bile in hamsters (12) or prevented formation of cholesterol monohydrate crystals in bile of prairie dogs (15). In healthy human subjects, ingestion of n-3 PUFAs (1.5 gm/day for $6 \mathrm{wk}$ ) lowered cholesterol saturation of bile (16). Therefore we studied in patients with cholesterol gallstones whether dietary supplementation with n-3 PUFAs decreases cholesterol saturation and prevents formation of cholesterol crystals in bile.

\section{SUBJECTS AND METHODS}

Subjects. Seven otherwise healthy patients (five women, two men; age range $=27$ to $56 \mathrm{yr}$ ) with symptomatic gallstones were studied (Table 1). All had radiolucent gallbladder stones and visible gallbladders on cholecystography. None had hypertriglyceridemia or a serum cholesterol level exceeding $260 \mathrm{mg} / \mathrm{dl}$ or were obese. None had a history of allergy to fish protein, excessive alcohol intake or intake (within the last $6 \mathrm{wk}$ ) of antibiotic drugs, laxative drugs or drugs interfering with lipid metabolism. The fish oil supplement was well tolerated except in one patient, who experienced uncharacteristic upper abdominal discomfort that lasted 5 days.

Study Protocol. The protocol had been approved $a$ priori by the ethics committee of this hospital. All participants gave written, informed consent. They were studied twice: first on their regular Western diet without supplementation (control study) and again after 5 wk of dietary supplementation with fish oil ( $\mathrm{n}-3$ PUFA study). All ingested $11.3 \mathrm{gm} /$ day MaxEPA oil (in three doses) (Eicosapen; generously provided by $\mathrm{Ny}$ comed Arzneimittel GmbH, Munich, Germany); this preparation consisted of triglycerides with a fatty acid pattern of $30 \%$ n-3 PUFAs (18\% [20:5] eicosapentaenoic and 12\% [22:6] docosahexaenoic acid), 21\% oleic acid (18:1, $\mathrm{n}=9$ PUFAs) and 
TABLE 1. Characteristics of participating gallstone patients

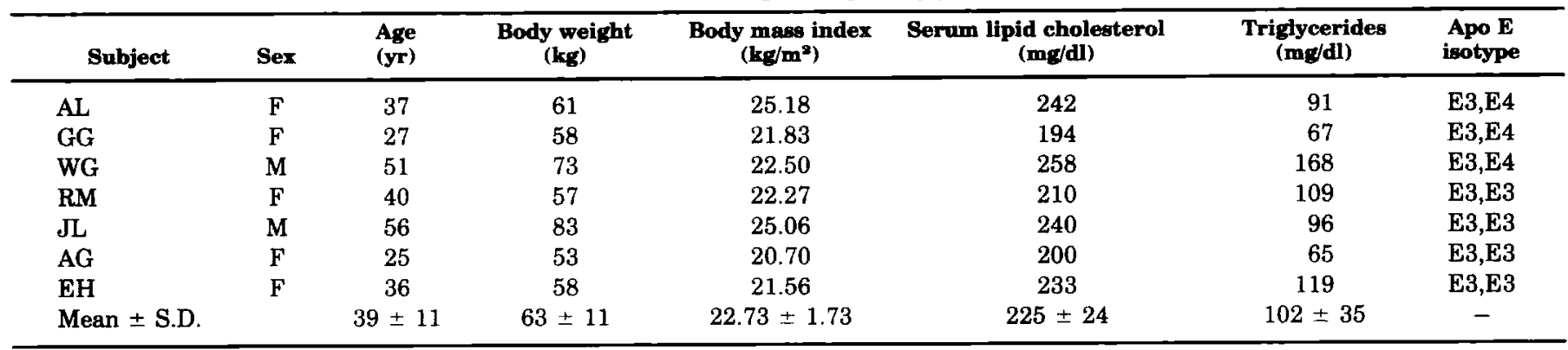

$28 \%$ saturated fatty acids, with small amounts of cholesterol $(0.2 \mathrm{mg} / \mathrm{gm})$ and vitamin $\mathrm{E}(2 \mathrm{mg} / \mathrm{gm})$. The daily dose of $\mathrm{n}-3$ PUFAs was $3.75 \mathrm{gm}$; that of eicosapentaenoic acid was $2 \mathrm{gm}$. A design detailed in a previous report (17) was used for control and n-3 PUFA studies. After patients fasted overnight (12 hr), duodenal bile was sampled, light-shielded, into an ice-cooled beaker through a nasoduodenal tube; gallbladder emptying was studied every 5 min on sonographic imaging (18) during intravenous infusion of cholecystokinin (CCK) $(0.02$ Ivy dog units $\cdot \mathrm{kg}^{-1} \cdot \mathrm{min}^{-1}$ for $40 \mathrm{~min}$ ) (19). Gallstone volume (V) was calculated from sonographic estimates of the maximum diameter $\left(d_{1}=2 a\right)$ and the maximal perpendicular diameter $\left(\mathrm{d}_{2}=2 \mathrm{~b}\right)$, assuming a three-dimensional ellipsoid $\left(V=\frac{4}{3} a ~ b^{2}\right)$. Bile was collected from 10 to $20 \mathrm{~min}$ and from 20 to $30 \mathrm{~min}$ during the CCK infusion, and the darker fraction was analyzed. In addition, $0.5-\mathrm{ml}$ specimens of fresh duodenal bile were sampled at $10,15,20$ and $30 \mathrm{~min}$ of CCK infusion for light microscopy.

Light Microscopy. The number of cholesterol monohydrate crystals per microliter was counted in fresh duodenal bile and in an immediately obtained sediment of $200 \mu \mathrm{l}$ bile (range of specific weight $=1.002$ to $1.044 \mathrm{gm} / \mathrm{ml} ; 10 \mathrm{~min}$ centrifugation at $11,000 \mathrm{~g}$ ). Cholesterol crystals were identified by their typical appearance on direct light microscopy and birefringence on polarized light microscopy $(20,21)$; they were immediately counted in a hemacytometer. The greatest number counted in the sediment at any time point was used for statistical analysis. The counting procedure for cholesterol crystals was reproducible in different aliquots of the same bile specimen (coefficient of variation $=10.1 \% ; \mathrm{n}=10$ ); recovery $=94 \% \pm 15 \%$ (mean \pm S.D.; $\mathrm{n}=10$ ) in sediments compared with the count in native bile (i.e., count of $0.1 \mu \mathrm{l}$ multiplied by factor 10 ).

Chemical Analysis of Bile Samples. The samples were kept in chloroform/methanol $(2: 1, \mathrm{vol} / \mathrm{vol})$ at $-20^{\circ} \mathrm{C}$ for analysis. Cholesterol (22) and lipid phosphorus (23) were measured with colorimetric tests; bile acid species were assayed with gasliquid chromatography (GLC) (24). For duodenal bile the cholesterol saturation index was calculated (25) according to the method of Carey and Small (26) on the basis of actual lipid concentrations. For comparison, the saturation index of duodenal bile was also calculated $(25,27)$ for a fixed lipid concentration of $10 \mathrm{gm} / \mathrm{dl}$, as assumed for undiluted gallbladder bile.

The fatty acid species of biliary phospholipids were analyzed with GLC (28). Total lipids were extracted from native bile (50 to $100 \mu \mathrm{l}$ with $20 \mu \mathrm{g}$ diheptadecanoyl phosphatidylcholine as internal standard) with chloroform/methanol and the phospholipid fraction isolated on Bondelut $\mathrm{C}_{18}$ cartridges (Waters Chromatography Division, Millipore Corp., Bedford, MA) (29). The phospholipid fraction was refluxed for $1 \mathrm{hr}$ in $3 \mathrm{~N}$ methanolic $\mathrm{HCl}$ at $75^{\circ} \mathrm{C}$ for acidic transmethylation of phospholipid fatty acids. The fatty acid methyl esters were extracted with hexane and analyzed with GLC on a cyanopropyl siloxane capillary column (CP-Sil 88; Chrompak, Middleburg, The Netherlands), which was used with hydrogen on a temperature program $\left(80^{\circ}\right.$ to $\left.200^{\circ} \mathrm{C}\right)$. Fatty acids were identified on retention time and cochromatography with authentic standards.

Determination of Nucleation Time. Time elapsed before in vitro appearance of cholesterol crystals from duodenal bile was determined by the method of Holan et al. (30) as modified by Marks et al. (31). Native duodenal bile ( $\geq 4 \mathrm{ml}$ ) was centrifuged for $2 \mathrm{hr}$ at $100,000 \mathrm{~g}$ and $25^{\circ} \mathrm{C}$ to obtain a crystal-free isotropic interphase of bile, as described by Holan et al. (30). An aliquot of the isotropic interphase was immediately examined microscopically to confirm the absence of crystals. An aliquot (500 $\mu$ ) of the isotropic interphase was added to tubes 1 and 2, which contained (a) $5 \mu \mathrm{l}$ sodium azide (20\%) and (b) a freshly prepared mixture $(25 \mu$ l) of the proteinase inhibitors PMSF (final concentration $=1 \mathrm{mmol} / \mathrm{L}$ ) and soybean trypsin inhibitor $(50 \mu \mathrm{g} / \mathrm{ml})$, the phospholipase inhibitor parabromophenacyl bromide $(0.4 \mathrm{mmol} / \mathrm{L})$ and antibiotics (streptomycin, $100 \mu \mathrm{g} / \mathrm{ml}$; penicillin, $100 \mathrm{U} / \mathrm{ml}$, respectively). Samples were incubated under nitrogen at $37^{\circ} \mathrm{C}$ in the dark. Each day for 21 days an aliquot ( 5 to $10 \mu \mathrm{l}$ ) of the incubated bile was examined for appearance of cholesterol crystals. Nucleation time as observed with incubate $b$ was recorded as the interval from the start of the incubation to the first appearance of cholesterol crystals in bile. If cholesterol crystals had not appeared after 21 days, bile was considered nonnucleating (recorded as nucleation time of 21 days).

The effect of pure L- $\alpha$-palmitoyl-eicosapentaenoyl phosphatidylcholine (Avanti Polar Lipids Inc., Alabaster, $\mathrm{AL}$ ) on in vitro nucleation time of cholesterol crystals was studied in surgical specimens of gallbladder bile obtained from four patients ( 3 women and 1 man; mean age $=46 \pm 11 \mathrm{yr}$ ) who had undergone cholecystectomy for radiolucent gallstones; all four native bile specimens had contained cholesterol crystals. Crystal-free, isotropic bile was obtained on ultracentrifugation. L- $\alpha$-palmitoyl-eicosapentaenoyl phosphatidylcholine $(10 \mathrm{mg} / \mathrm{ml}$ chloroform) was added in appropriate amounts to the test tubes, dried under a stream of nitrogen and redissolved in $0.30 \mathrm{ml}$ isotropic bile. Complete dissolution was achieved in the samples, as documented by analysis of the concentration and the fatty acid pattern of phospholipids. Aliquots of isotropic bile $(0.30 \mathrm{ml}$ with $0.1 \% \mathrm{vol} / \mathrm{vol}$ sodium azide) were incubated in native form (30) and after addition of $1.0 \mu \mathrm{mol}$ and $1.5 \mu \mathrm{mol}$, respectively, of $\mathrm{L}-\alpha$-palmitoyl-eicosapentaenoyl phosphatidylcholine under nitrogen at $37^{\circ} \mathrm{C}$ in the dark and examined daily for appearance of cholesterol crystals.

Serum Lipid Profiles. Serum samples obtained after $12 \mathrm{hr}$ 


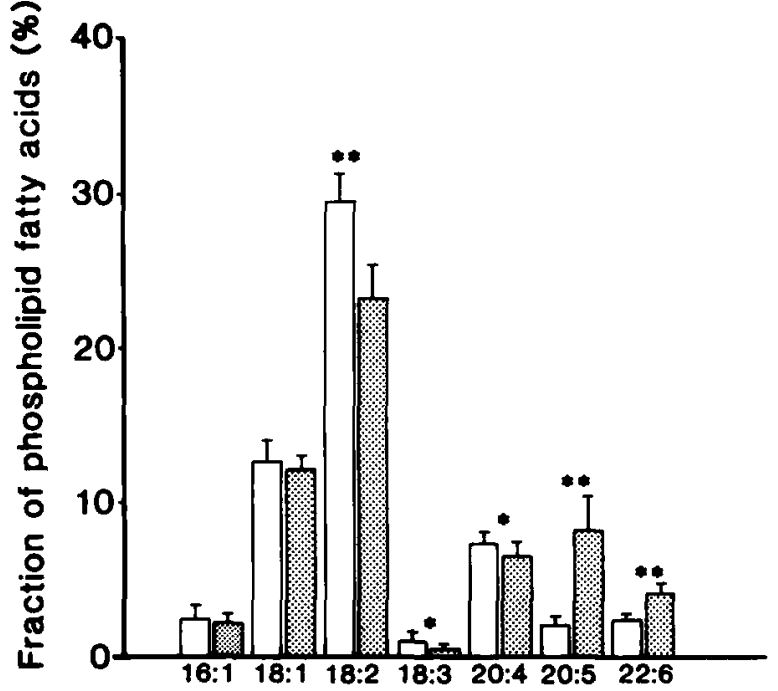

Unsaturated fatty acids of biliary phospholipids

Fig. 1. Effect of dietary n-3 PUFAs ( $3.75 \mathrm{gm} /$ day for $5 \mathrm{wk}$ ) on unsaturated fatty acids of biliary phospholipids in seven gallstone patients. Eicosapentaenoic acid $(20: 5, n-3)$ and docosahexaenoic acid $(22: 6, n-3)$ of dietary origin were incorporated into phospholipids of bile, where they partly displaced linoleic acid $(18: 2, n-6), \alpha$-linolenic acid $(18: 3, n-3)$ and arachidonic acid $(20: 4, n-6)$. The fractions of palmitoleic acid (16:1) and oleic acid (18:1) did not change. Data expressed as mean \pm S.D. ${ }^{*} p<0.02 ;{ }^{* *}$ p $<0.001 ; \square=$ control study; 国-3 PUFA study.

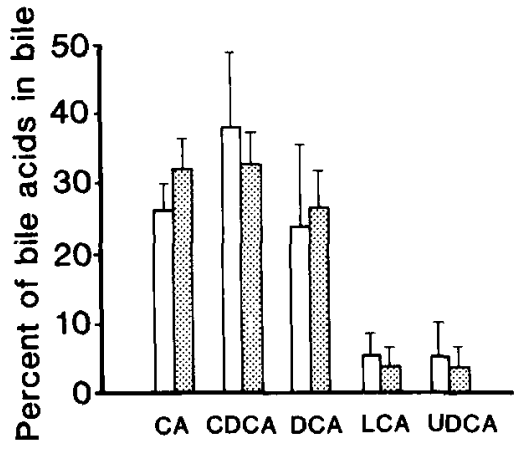

FIG. 2. Pattern of biliary bile acids in seven gallstone patients before ( $\square$ ) and after (18) intake of a fish oil supplement (3.75 g n-3 PUFAs/day) for $5 \mathrm{wk}$. The fraction of cholic acid increased at the expense of chenodeoxycholic acid. The pattern of secondary bile acids did not change. Data expressed as mean \pm S.D. ${ }^{*} \mathrm{p}=0.07$. CA $=$ cholic acid; CDCA = chenodeoxycholic acid; DCA $=$ deoxycholic acid; LCA = lithocholic acid; UDCA = ursodeoxycholic acid.

fasting before and during intake of n-3 PUFAs were analyzed enzymatically for cholesterol and triglycerides in total serum and in the very low density lipoprotein (VLDL) and highdensity lipoprotein (HDL) fractions (32). For analysis of lipoprotein classes, the VLDL fraction was separated by ultracentrifugation (at density $\mathrm{d}=1.006 \mathrm{gm} / \mathrm{ml}$ at $50,000 \mathrm{rpm}$ for $20 \mathrm{hr}$ at $4^{\circ} \mathrm{C}$ in a Beckman Ti 50 rotor (Beckman Instruments, Inc., Fullerton, CA) (33). HDL cholesterol was measured in the bottom fraction after precipitation of lowdensity lipoprotein (LDL) with phosphotungstic acid. LDL cholesterol concentration was calculated by subtracting HDL cholesterol from total cholesterol concentration of the bottom
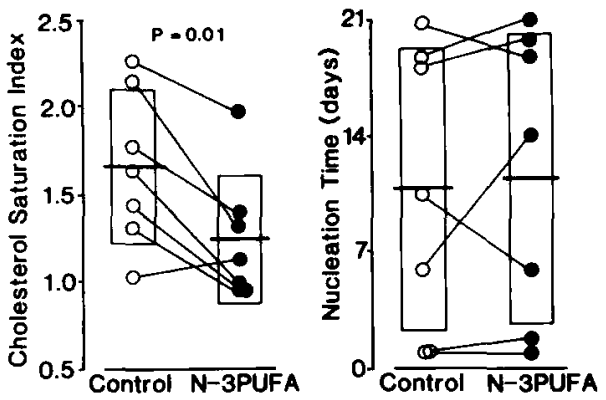

FIG. 3. Effect of dietary n-3 PUFAs ( $3.75 \mathrm{gm} /$ day for $5 \mathrm{wk}$ ) on cholesterol saturation index (26) and on in vitro nucleation time of cholesterol crystals (31) in CCK-stimulated duodenal bile of seven gallstone patients. The average decrease of cholesterol saturation by $25 \%$ conformed to a decrease by $19 \%$ in the molar ratio of cholesterol to phospholipids. Nucleation time remained unchanged. Data expressed as mean \pm S.D.

fraction (32, 33). The apolipoprotein $\mathrm{E}$ isotype (33) was determined before patients' intake of $n-3$ PUFAs.

Statistical Analysis. Statistical significance of differences between groups was calculated with Student's $t$ test for paired observations after testing of the equality of variances with an F test and the Wilcoxon matched-pair, signed-rank test (34). All results, unless stated otherwise, are expressed as mean \pm S.D. $p<0.05$ was considered statistically significant.

\section{RESULTS}

Serum Lipid Concentrations. Intake of fish oil decreased ( $p<0.01$ ) triglyceride concentration in serum by $26 \%$ (from $102 \pm 25$ to $75 \pm 23 \mathrm{mg} / \mathrm{dl}$ ), and triglyceride concentration in VLDLs by $44 \%$ (from $66 \pm 36$ to $36 \pm 21 \mathrm{mg} / \mathrm{dl}$ ) and decreased the VLDL cholesterol level by $41 \%$ (from $17 \pm 9$ to $10 \pm 5 \mathrm{mg} / \mathrm{dl}$ ). The concentrations of total cholesterol $(+2 \%)$ and LDL cholesterol $(+2 \%)$ remained unchanged, whereas HDL cholesterol level showed a trend $(p=0.08)$ toward increase (from $52 \pm 15$ to $61 \pm 23 \mathrm{mg} / \mathrm{dl}$ ).

Fatty Acid Composition of Biliary Phospholipids. Dietary n-3 PUFAs were incorporated into biliary phospholipids. The average fraction of eicosapentaenoic acid increased from $2.0 \% \pm 0.6 \%$ to $8.2 \% \pm 2.2 \%$ and that of docosahexaenoic acid increased from $2.4 \% \pm 0.4 \%$ to $4.2 \% \pm 0.6 \%$ ( $p<0.001$ for both) during intake of fish oil, whereas $\alpha$-linolenic acid (18:3) $(1.0 \% \pm 0.6 \%$ to $0.5 \% \pm 0.3 \%)$ and the n-6 PUFAs linoleic acid $(18: 2)(29 \% \pm 2 \%$ to $23 \% \pm 2 \%)$ $(\mathrm{p}<0.001)$ and (to a lesser extent) arachidonic acid $(20: 4)(7.3 \% \pm 0.7 \%$ to $6.5 \% \pm 0.9 \% ; p<0.02)$ were partly replaced (Fig. 1). The fractions of palmitic acid $(16: 0)(33 \% \pm 3 \%)$, stearic acid $(18: 0)(5.6 \% \pm 1.1 \%)$ and palmitoleic acid $(16: 1, n-7)(2.5 \% \pm 0.8 \%)$ did not change significantly during intake of fish oil.

Composition of Biliary Bile Acids. During intake of fish oil the fraction of cholic acid in biliary bile acids increased from $26.3 \% \pm 4.0 \%$ to $32.3 \% \pm 4.3 \%(\mathrm{p}=$ 0.07 ), and the ratio of cholic acid to chenodeoxycholic acid increased from $0.72 \pm 0.16$ to $1.02 \pm 0.28(\mathrm{p}=$ 0.01 ). The fraction of chenodeoxycholic acid decreased on average by $5.5 \%$ (NS). The fractions of secondary 
TABLE 2. Effect of fish oil intake on cholesterol content and nucleation of cholesterol monohydrate crystals in CCK-stimulated bile

\begin{tabular}{|c|c|c|c|c|c|c|c|c|}
\hline Subject & \multicolumn{2}{|c|}{$\begin{array}{c}\text { Total lipids } \\
\text { (gm/dl) }\end{array}$} & \multicolumn{2}{|c|}{ Cholesterol saturation index ${ }^{a}$} & \multicolumn{2}{|c|}{$\begin{array}{c}\text { Nucleation time } \\
\text { days }\end{array}$} & \multicolumn{2}{|c|}{$\begin{array}{l}\text { Cholesterol crystals } \\
\text { (count } / \mu 1)\end{array}$} \\
\hline $\mathrm{AL}$ & 2.90 & 2.09 & 2.26 & 1.97 & 6.0 & 13.8 & 9 & 54 \\
\hline GG & 1.17 & 5.03 & 1.31 & 0.92 & 10.0 & 4.8 & 0 & 202 \\
\hline WG & 1.52 & 4.10 & 1.78 & 1.41 & 0.8 & 1.4 & 0 & 30 \\
\hline $\mathbf{R M}$ & 1.36 & 5.17 & 1.66 & 0.99 & 17.9 & 19. & 1 & 253 \\
\hline JL & 3.08 & 2.80 & 1.44 & 0.92 & 19.0 & 21.0 & 10 & 0 \\
\hline Mean \pm S.D. & $2.35 \pm 1.01$ & $3.49 \pm 1.27$ & $1.60 \pm 0.44$ & $1.24 \pm 0.38$ & $10.8 \pm 8.6$ & $11.4 \pm 8.8$ & $10 \pm 15$ & $85 \pm 100$ \\
\hline
\end{tabular}

${ }^{a}$ Calculated from actual lipid concentrations according to the method of Carey and Small (26). Cholesterol saturation decreased by $20 \%$ (from $1.27 \pm 0.34$ to $1.01 \pm 0.27[\mathrm{p}=0.02]$ ) when calculated from relative lipid content (27) assuming a fixed total lipid concentration of 10 gm/dl for undiluted bile.

bile acids deoxycholic acid, lithocholic acid and ursodeoxycholic acid did not change significantly (Fig. 2).

Composition of Biliary Lipids. The cholesterol saturation index (26) decreased by $25 \%$, from $1.66 \pm 0.44$ to $1.24 \pm 0.38(\mathrm{p}=0.01)$ (Table 2; Fig. 3), and the molar ratio of cholesterol to phospholipids decreased by $19 \%$ ( $p<0.05$ ) during intake of fish oil; the ratio of phospholipids to bile acids did not change. Bile acid concentration ( $26 \pm 12$ vs. $40 \pm 18 \mathrm{mmol} / \mathrm{L})$ and total lipid content $(2.4 \pm 0.9$ vs. $3.5 \pm 1.3 \mathrm{gm} / \mathrm{ml})$ (Table 2$)$ of CCK-stimulated duodenal bile were increased on average by $50 \%$ and $43 \%$ (NS), respectively.

Cholesterol Monohydrate Crystals in Bile. CCKstimulated duodenal bile showed cholesterol monohydrate crystals in five of seven patients during the control study and in six of seven patients during fish oil intake. Three subjects (GG, WG, RM) had at least 10 times more cholesterol crystals and a marked increase (2.7-fold to 4.3-fold) of total lipid concentration in bile; two (WG, RM) had also emptied a higher fraction of gallbladder volume during fish oil intake. The increase of the average count of cholesterol crystals in bile during ingestion of fish oil was not statistically significant $(\mathrm{p} \geq 0.10$ ). The count of cholesterol crystals was correlated neither with the nucleation time of duodenal bile $(r=0.07)$ nor with the cholesterol saturation index $(\mathbf{r}=-0.35)$.

Cholesterol Crystal Nucleation Time. In vitro, the nucleation time of duodenal bile was not changed by intake of fish oil (Table 2; Fig. 3). Nucleation time of duodenal bile was correlated in an inverse linear fashion with the cholesterol saturation index $(\mathrm{r}=-0.45$; $p=0.08$ ), but not with the count of cholesterol monohydrate crystals $(r=0.07 ; \mathrm{NS})$.

When pure palmitoyl-eicosapentaenoyl-phosphatidylcholine was added in vitro to surgical specimens of isotropic, supersaturated gallbladder bile from four patients with cholesterol gallstones in amounts that increased the eicosapentaenoic acid fraction of the phospholipids, but barely decreased cholesterol saturation, the nucleation time for cholesterol crystals was prolonged (Table 3 ).
Gallbladder Emptying by CCK. The volume of the gallbladder in the fasting state was not different before and during intake of fish oil. Impaired emptying of the gallbladder in response to the CCK infusion (i.e., emptying of less than $50 \%$ of the fasting volume [19]), was not improved in two gallstone patients (AL, GG); nor was normal emptying (in JL, AG, EH) impaired by ingestion of fish oil. Only two patients (WG, RM) with emptying in the lower range of normal had higher fractional emptying of the gallbladder during intake of fish oil (Table 4). In the entire group, the time course of gallbladder emptying (Fig. 4) and the fraction of the gallbladder volume emptied (Table 4) were very similar before and during intake of fish oil.

\section{DISCUSSION}

Supplementation of a Western diet with marine fish oil rich in n-3 PUFAs ( $3.75 \mathrm{gm} /$ day) markedly increased the fraction of the n-3 PUFAs eicosapentaenoic acid $(20: 5 ; n-3)$ and docosahexaenoic acid $(22: 6 ; n-3)$ and replaced about one third of the linoleic acid $(18: 2 ; n-6)$ in the phospholipids of bile. The cholesterol/phospholipid molar ratio and cholesterol saturation decreased by $19 \%$ and $25 \%$, respectively, whereas the nucleation time of duodenal bile did not change. But this neither prevented precipitation of cholesterol monohydrate crystals in bile nor improved emptying of the gallbladder in gallstone patients.

This raises the question of whether patients were given adequate doses of n-3 PUFAs. Doses in the range of $2 \mathrm{gm}$ to $20 \mathrm{gm}$ of n-3 PUFAs/day (as marine fish oil) have been used to reduce triglyceride and VLDL concentrations in serum (35-40) by decreasing hepatic secretion of VLDL (38). In moderate hypertriglyceridemia, the maximum effect on triglyceride levels had already been achieved with a daily dose of 3 to $5 \mathrm{gm} \mathrm{n-3}$ PUFAs and was characterized by an approximate 50\% lowering of VLDL triglycerides and VLDL cholesterol, little change in LDL cholesterol level and a small decrease (35-38) or small increase $(39,40)$ of $\mathrm{HDL}$ cholesterol. In our participants, who all had normotriglyceridemia, these effects on serum lipid levels were 
TABLE 3. Effect in vitro of 16:0,20:5-phosphatidylcholine on nucleation time of gallbladder bile samples

\begin{tabular}{|c|c|c|c|c|c|}
\hline \multirow{2}{*}{$\begin{array}{c}\text { Amount of added } \\
16: 0,20: 5 \text {-phosphatidylcholine } \\
\text { ( } \mu \mathrm{mol} / 0.30 \mathrm{ml} \text { bile })\end{array}$} & \multirow{2}{*}{$\begin{array}{l}\text { Total lipids } \\
\text { (gm/dl) }\end{array}$} & \multirow{2}{*}{$\begin{array}{c}\text { Cholesterol } \\
\text { saturation index }\end{array}$} & \multicolumn{2}{|c|}{ Phospholipid fatty acids (\%) } & \multirow{2}{*}{$\begin{array}{c}\text { Nucleation time } \\
\text { (days) }\end{array}$} \\
\hline & & & Eicosapentaenoic & Linoleic & \\
\hline $\mathbf{0}$ & $9.8 \pm 5.9^{a}$ & $1.16 \pm 0.23$ & $1.7 \pm 0.9$ & $28.2 \pm 2.3$ & $2.7 \pm 2.4$ \\
\hline 1.5 & $10.3 \pm 6.0$ & $1.10 \pm 0.17$ & $7.6 \pm 5.2$ & $24.8 \pm 2.5$ & $16.1 \pm 8.3$ \\
\hline
\end{tabular}

Data expressed as mean \pm S.D. $(n=4)$.

TABLE 4. Gallbladder emptying by intravenous $\mathrm{CCK}^{\alpha}$

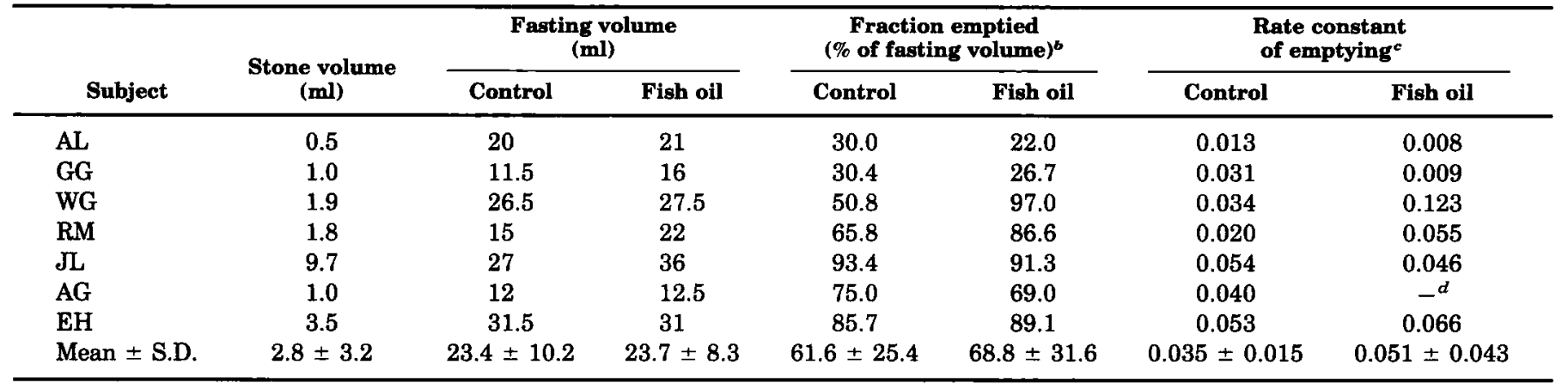

${ }^{a}$ Determined by serial sonographic imaging and calculation of volume of the gallbladder during intravenous infusion of CCK ( 0.02 Ivy dog units $\cdot \mathrm{kg}^{-1} \cdot \mathrm{min}^{-1}$ for $40 \mathrm{~min}$ ).

${ }^{b}$ The estimated stone volume had been subtracted from the fasting volume and from the residual volume (after 40-min CCK infusion); the fraction emptied (\%) was then calculated as $100 \times\left(\mathrm{V}_{\text {corrected fasting }}-\mathrm{V}_{\text {corrected residual }}\right) / \mathrm{V}_{\text {corrected fasting. }}$

'Slope of the first-order linear function of gallbladder volumes vs. time (18).

${ }^{d}$ Bile was sampled during upper gastrointestinal endoscopy; only fasting and residual volumes of the gallbladder were determined.

reproduced by a dose of $3.75 \mathrm{gm} /$ day of $\mathrm{n}-3$ PUFAs, confirming adequate dosage and good compliance.

The dose of fish oil $(11.3 \mathrm{gm} /$ day $=3.75 \mathrm{gm} /$ day $\mathrm{n}-3$ PUFAs) administered in our study displaced about one third of the linoleic acid, the major unsaturated fatty acid of biliary phospholipids, but only very small amounts of arachidonic acid and none of the oleic acid (Fig. 1). Unsaturated phospholipid fatty acids in bile predominantly represent distinct phosphatidylcholine species because at least $95 \%$ of biliary phospholipids are phosphatidylcholines and the unsaturated fatty acids of biliary phosphatidylcholines are located in position sn-2 (except for some 3\% to 5\% of oleic acid that resides in position sn-1 of 18:1-18:2 phosphatidylcholine) (7). The most abundant fatty acid in position sn-2 is linoleic acid $(18: 2, n-6)$, primarily in $16: 0,18: 2$-phosphatidylcholine (7). Dietary n-3 PUFAs displaced mainly 18:2containing phosphatidylcholines with 20:5-containing and 22:6-containing phosphatidylcholines. Several factors may contribute to this rather selective exchange. (a) Highly unsaturated fatty acids are preferred over monounsaturated or diunsaturated fatty acids for the sn-2 position of phosphatidylcholines destined for secretion into bile $(41,42)$. This could explain why dietary n-3 PUFAs displaced linoleic acid rather than arachidonic acid in biliary phospholipids. (b) Similar changes of the fatty acid pattern with poor displacement of arachidonic acid by n-3 PUFAs have been observed in serum phospholipids in response to fish oil intake (43). (c) The magnitude and selectivity of displacement of fatty acid species could also depend on the magnitude of the respective fatty acid fraction in biliary phosphatidylcholines before fish oil intake and on the entire fatty acid spectrum of the ingested fish oil. Because the gallstone patients in this study had normal fractions of arachidonic acid, not increased fractions (44), the fraction of displaceable arachidonyl lecithins may have been low before intake of fish oil. Furthermore, the fish oil contained only $29 \%$ of fatty acids as n-3 PUFAs, but some $21 \%$ as oleic acid (18:1, n-9). Failure to substitute any of the oleic acid located mainly in 16:0, 18:1phosphatidylcholines (7) by n-3 PUFAs may be related to the considerable dose of oleic acid contained in the fish oil preparation.

A 25\% decrease in cholesterol saturation of bile was observed during intake of fish oil (Fig. 3). This was caused entirely by a decrease in the cholesterol/phospholipid molar ratio; the phospholipid/bile acid molar ratio remained constant. This decrease in cholesterol/phospholipid molar ratio cannot be attributed to the small change of the bile acid pattern. How, then, can the decrease by n-3 PUFAs of the cholesterol/phospholipid ratio in bile be explained?

Dietary n-3 PUFAs could have reduced the amount of free cholesterol available in the liver for biliary secretion. One study has revealed that excess cholesterol secreted in bile of subjects with cholesterol gallstones can result from inappropriately high synthesis of cholesterol in the liver (1). As a consequence, inhibition of cholesterol synthesis should decrease biliary secretion of cholesterol. Dietary n-3 PUFAs suppress the rate of hepatic synthesis of cholesterol in the rat (45), but it has 
not yet been studied whether they have the same effect in human beings.

Alternatively, the cholesterol/phospholipid ratio in bile could have been lowered by the changes in biliary phosphatidylcholine species during intake of fish oil. In a previous study in healthy women and cholesterol gallstone patients we observed positive linear correlations between the cholesterol saturation index (and the cholesterol/phospholipid ratio) and the fractions of oleic acid (18:1) and arachidonic acid (20:4) and an inverse relationship between the saturation index and the fraction of linoleic acid (18:2), palmitoleic acid (16:1) and eicosapentaenoic acid (20:5) in biliary phospholipids (44). These correlations could represent differences among the respective biliary phosphatidylcholine species in cholesterol/phospholipid packing into the membrane of biliary vesicles. In this case, eicosapentaenoic acidcontaining phosphatidylcholine would pack less cholesterol into secretory vesicles than, for instance, phosphatidylcholines containing arachidonic acid, oleic acid or even linoleic acid. The moderate decrease in cholesterol saturation during intake of fish oil could be explained by the fact that n-3 PUFAs displaced phosphatidylcholine containing linoleic acid, which also is associated with low cholesterol saturation $(7,44)$, rather than phosphatidylcholines containing arachidonic acid or oleic acid. Also in the hamster, the secretory ratio of cholesterol to phospholipids in bile was decreased when linoleic acid in biliary phosphatidylcholines was displaced in part with n-3 PUFAs of dietary origin (12). A study in African green monkeys that was published after conclusion of this study showed similar effects. Feeding of a fish-oil-supplemented diet for $2 \frac{1 / 2}{2}$ to $3 \mathrm{yr}$ (compared with a lard-supplemented diet) led to a similar modification of phospholipid fatty acids and to $25 \%$ lower cholesterol saturation of bile without significant differences in biliary bile acids. The fish oil diet was also associated with a lower rate of gallstone formation (22\% vs. $67 \%$ ) (13).

$\mathrm{L}-\alpha-16: 0,20: 5-$ phosphatidylcholine, which is the phosphatidylcholine species mainly increased in bile by dietary n-3 PUFAs, retards nucleation of cholesterol crystals (Table 3 ) when added in vitro to supersaturated gallbladder bile in proportions similar to those found in duodenal bile during fish oil intake (compare Fig. 1). However, the change in phosphatidylcholine fatty acids induced by dietary n-3 PUFAs did not prolong nucleation time of duodenal bile in our patients (Fig. 3). Certainly nucleation time is longer in duodenal bile than in gallbladder bile (31); therefore further prolongation could be difficult to detect in duodenal bile. In addition, other factors could have contributed to this apparent lack of effect on native duodenal bile. The $49 \%$ average increase in lipid concentration during intake of fish oil could have offset prolongation of nucleation time; according to Marks et al. (31), nucleation time is shorter by $31 \%$ in bile with a high lipid concentration (11.3 $\mathrm{gm} / \mathrm{dl}$ ) than in diluted bile (lipids, $7.6 \mathrm{gm} / \mathrm{dl}$ ). Furthermore, during intake of fish oil 16:0,20:5phosphatidylcholine might have displaced another phos-

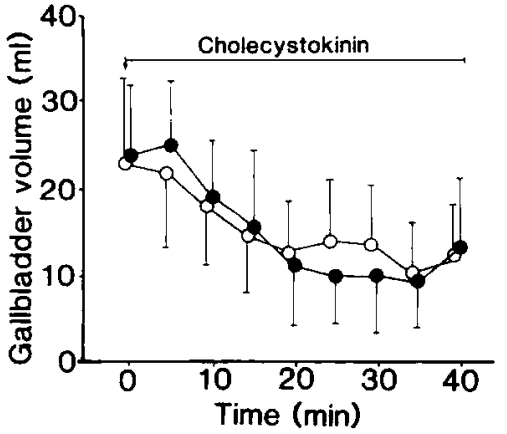

FIG. 4. Gallbladder emptying during intravenous infusion of cholecystokinin $\left(0.02\right.$ Ivy dog units $\mathrm{kg}^{-1} \cdot \min ^{-1}(18,19)$ in six gallstone patients before $(0=$ control) and after intake for 5 wk of marine fish oil at a daily dose of 3.75 gm n-3 PUFAs $(\bullet=$ on $n-3$ PUFA). No significant differences in gallbladder emptying were seen. Data expressed as mean \pm S.D.

phatidylcholine species with similar retardant effect on cholesterol nucleation, whereas during the in vitro experiment the concentrations of all other phosphatidylcholine species remained nearly constant. Presumably 16:0,20:5-phosphatidylcholine mainly displaced 16:0,18:2-phosphatidylcholine (see Fig. 1), another hydrophilic phosphatidylcholine species, which facilitates micellar dissolution of multilamellar cholesterol/phosphatidylcholine vesicles by bile salts (46) and so could prolong the time for nucleation of cholesterol crystals.

The count of cholesterol crystals in duodenal bile (Table 2) appears to bear qualitative (presence vs. absence) rather than quantitative significance because the number of crystals counted was correlated neither with the nucleation time nor with the cholesterol saturation index of bile. This raises the possibility that the crystal count in duodenal bile depends on the expulsion of cholesterol crystals from the gallbladder. The expulsion of crystals may have been facilitated during fish oil intake (e.g., by decreased adherence to the mucin layer of the gallbladder) or, in two patients (WG, RM), by enhanced gallbladder emptying.

The change in phosphatidylcholine fatty acids induced by dietary n-3 PUFAs did not prevent formation of cholesterol crystals in supersaturated bile of gallstone patients (Table 2). This observation is in agreement with the lack of effect on the nucleation time of duodenal bile. By contrast, in the prairie dog model of gallstone formation dietary fish oil prevented precipitation of cholesterol crystals in supersaturated gallbladder bile (15). Which factors could have contributed to the different responses in human subjects and prairie dogs? First, in the prairie dog model dietary n-3 PUFAs may have interfered with prostaglandin formation (15). Dietary n-3 PUFAs can decrease formation of prostaglandins $\mathrm{F}_{2 \alpha}$ and $\mathrm{E}_{2}$ by substituting as substrate eicosapentaenoic $(20: 5, \mathrm{n}-3)$ for arachidonic acid $(20: 4$, $\mathrm{n}-6)$ (47). Inhibition of prostaglandin synthesis in gallbladder tissue by aspirin prevented hypersecretion of mucous glycoproteins and formation of cholesterol 
crystals in the gallbladder of the cholesterol-fed prairie dog in an earlier study (48), but not in recent studies (49, 50). In any case, our human study probably interfered little with prostaglandin formation in the gallbladder because very little arachidonic acid had been displaced by eicosapentaenoic acid and docosahexaenoic acid in biliary phospholipids. Second, in the prairie dog dietary n-3 PUFAs prevented crystal formation in the absence of gallstones (15), whereas in our human study gallstones had already been present. The high coincidence $(80 \%$ to $100 \%$ ) of cholesterol gallstones and presence of cholesterol crystals in gallbladder bile $(21,51,52)$ raises the possibility that these crystals nucleated on the free cholesterol surfaces of the stones and were shed into bile. During fish oil intake cholesterol crystals may have been detached more easily by the surfactant effects of the more hydrophilic phosphatidylcholines. Third, increasing the bile acid concentration of supersaturated bile in the gallbladder enhances formation of very cholesterol rich phospholipid vesicles, which precede formation of cholesterol crystals (53). This phenomenon could contribute to formation of cholesterol crystals in saturated bile during intake of fish oil. Thus only those patients who had high increases in lipid and bile acid concentration during fish oil intake showed striking increases in crystal count.

In summary, supplementation of a Western diet with fish oil rich in $n-3$ PUFAs $(3.75 \mathrm{gm} /$ day) raised the content of the n-3 PUFAs eicosapentaenoic acid and docosahexaenoic acid in biliary phosphatidylcholines and partly displaced linoleic acid-containing phosphatidylcholine species in bile. The arachidonic acid fraction of biliary phosphatidylcholines, which had been in the normal range in these gallstone patients, decreased very little during intake of fish oil. These changes in fatty acids of biliary phospholipids were associated with a lower cholesterol/phospholipid molar ratio and cholesterol saturation of bile, most likely caused by lecithin species-dependent coupling of cholesterol for secretion. However, intake of n-3 PUFAs did not protect gallstone patients from nucleation of cholesterol crystals in supersaturated gallbladder bile; nor did it improve emptying of the gallbladder. Therefore the effects of fish oil in our study are not sufficient to recommend its use for medical treatment or prophylaxis of gallstones.

Acknowledgments: We thank Ms. Brigitte Ruhland, Mrs. Benedikta Zündt and Mrs. Margit Judenberg for technical assistance and Mr. Martin Mayer for microscopy of bile samples. We are grateful to Dr. Jay W. Marks (Cedars-Sinai Medical Center, UCLA School of Medicine, Los Angeles) for communicating the instructions for the nucleation time assay of duodenal bile.

\section{REFERENCES}

1. Holzbach RT. Pathogenesis and medical treatment of gallstones In: Sleisenger MH, Fordtran JS, eds. Gastrointestinal disease. Philadelphia: W.B. Saunders Co. 1989:1668-1690.

2. Hofmann AF. Medical treatment of cholesterol gallstones by bile desaturating agents. HEPATOLOGY 1984;4:199-208.

3. Carulli N, Loria P, Bertolotti M, Ponz de Leon M, Menozzi D,
Medici G, Piccagli I. Effects of acute changes of bile acid pool composition on biliary lipid secretion. J Clin Invest 1984;74: 614-624.

4. Wagner CI, Trotman BW, Soloway RD. Kinetic analysis of biliary lipid excretion in man and dog. J Clin Invest 1976;57:473-477.

5. Coleman R. Biochemistry of bile secretion (Review). Biochem J 1987;244:249-261.

6. Sömjen GJ, Gilat TA. A nonmicellar mode of cholesterol transport in human bile. FEBS Lett 1983;156:266-268.

7. Ahlberg J, Curstedt T, Einarsson K, Sjövall J. Molecular species of biliary phosphatidylcholines in gallstone patients: the influence of treatment with cholic acid and chenodeoxycholic acid. J Lipid Res 1981;22:404-409.

8. Glenn JL, Christens F, Dam H. Effects of dietary fats on the fatty acid composition of liver and bile phosphatides of hamsters. Biochim Biophys Acta 1964;84:753-755.

9. Dam H, Kruse I, Krogh Jensen M, Kallehauge ME. Studies on human bile. II. Influence of two different fats on the composition of human bile. Scand J Clin Lab Invest 1967;19:367-378.

10. Paul R, Ramesha CS, Ganguly J. On the mechanism of hypocholesterolemic effects of polyunsaturated lipids. Advances Lipid Res 1980;17:155-171.

11. Balasubramaniam SB, Simons LA, Chang S, Hickie JB. Reduction in plasma cholesterol and increase in biliary cholesterol by a diet rich in n-3 fatty acids in the rat. J Lipid Res 1985;26:684689.

12. Berr F, Schreiber E, Goetz A, Paumgartner G. Effect of dietary fatty acids on biliary lipid secretion in the hamster [Abstract]. J Hepatol 1989;9:8.

13. Scobey MW, Johnson FL, Parks JS, Rudel LL. Dietary fish oil effects on biliary lipid secretion and cholesterol gallstone formation in the African green monkey. HEPATOLOGY 1991;14: 679-684.

14. Kelly GJ. From bioenergetics to membrane protein structure and gene expression: formation and fates of plant polyunsaturated fatty acids. Trends Biochem Sci 1984;9:501-503.

15. Magnuson TH, Lillemoe KD, High RC, Pitt HA. Fish oil prevents cholesterol crystal nucleation and gallstone formation [Abstract]. Gastroenterology 1989;96:A625.

16. Wechsler JG, Swobodnik W, Wenzel H, Saal D, Janowitz P, Ditschuneit $H$. Einfluß von Omega-3-Fettsäuren auf biliäre Lipide und Lithogenität. Z Gastroenterol 1988;27:254-257.

17. Berr F, Stellaard F, Pratschke E, Paumgartner G. Effects of cholecystectomy on the kinetics of primary and secondary bile acids. J Clin Invest 1989;83:1541-1550.

18. Everson GT, Braverman DZ, Johnson ML, Kern F. A critical evaluation of real-time ultrasonography for the study of gallbladder volume and contraction. Gastroenterology 1980;79:40-46.

19. Pomeranz IS, Shaffer EA. Abnormal gallbladder emptying in a subgroup of patients with gallstones. Gastroenterology 1985;88: 787-791.

20. Juniper $\mathrm{K}$ Jr, Burson EN Jr. Biliary tract studies. II. The significance of biliary crystals. Gastroenterology 1957;32:175-211.

21. Sedaghat A, Grundy SM. Cholesterol crystals and the formation of cholesterol gallstones. N Engl J Med 1980;302:1274-1277.

22. Abell LL, Levy BB, Brodie BB, Kendall FE. A simplified method for the estimation of total cholesterol in serum and demonstration of its specificity. J Biol Chem 1952;195:357-359.

23. Barlett GR. Phosphorus assay in column chromatography. J Biol Chem 1959;234:466-468.

24. Berr F, Stellaard F, Goetz A, Hammer C, Paumgartner G. Ethinylestradiol stimulates a biliary cholesterol-phospholipid cosecretion mechanism in the hamster. HEPATOLOGY 1988;8: 619-624.

25. Kuroki S, Cohen BI, Carey MC, Mosbach EH. Rapid computation with the personal computer of the percent cholesterol saturation of bile samples. J Lipid Res 1986;27:442-446.

26. Carey MC, Small DM. Physical chemistry of cholesterol solubility in bile. Relationships to gallstone formation and dissolution in man. J Clin Invest 1978;61:998-1026.

27. Carey MC. Critical tables for calculating the cholesterol saturation of native bile. J Lipid Res 1978;19:945-955.

28. von Schacky C, Fischer S, Weber PC. Long-term effects of dietary 
marine $n-3$ fatty acids upon plasma and cellular lipids, platelet function and eicosanoid formation in humans. J Clin Invest 1985;76:1626-31.

29. Kaludzny MA, Duncan LA, Merritt MV, Epps DE. Rapid separation of lipid classes in high yield and purity using bonded phase columns. J Lipid Res 1985;26:135-140.

30. Holan KR, Holzbach RT, Hermann RE, Cooperman AM, Claffey WJ. Nucleation time: a key factor in the pathogenesis of cholesterol gallstone disease. Gastroenterology 1979;77:611617.

31. Marks JW, Broomfield P, Bonorris GG, Schoenfield LJ. Factors affecting the measurement of cholesterol nucleation in human gallbladder and duodenal bile. Gastroenterology 1991;101: 214-219.

32. Lippel K, ed. Manual of laboratory operations: lipids and lipoprotein analysis: Lipid Research Clinies Program, 1974. DHEW publication no. (NIH) 75-628. Washington, D.C.: United States Government Printing Office, 1974.

33. Parhofer KG, Richter WO, Schwandt P. Apolipoprotein E phenotype frequency in type II diabetic patients with different forms of hyperlipoproteinemia. Horm Metab Res 1990;22:589-594.

34. Snedecor GW, Cochran WG. Statistical methods. 7th ed. Ames, IA Iowa State University Press, 1980.

35. Harris WS, Connor WE, McMurry MP. The comparative reductions of the plasma lipids and lipoproteins by dietary polyunsaturated fats: salmon oil versus vegetable oils. Metabolism 1983; 32:179-184.

36. Phillipson BE, Rothrock DW, Connor WE, Harris ES, Illingworth DR. Reduction of plasma lipids, lipoproteins and apoproteins by dietary fish oils in patients with hypertriglyceridemia. N Engl J Med 1985;312:1210-1216.

37. Nestel PJ. Polyunsaturated fatty acids (n-3, n-6) (Review). Am J Clin Nutr 1987;45:1161-1167.

38. Nestel PJ, Connor WE, Reardon MF, Connor S, Wong S, Boston R. Suppression by diets rich in fish oil of very low density lipoprotein production in man. J Clin Invest 1984;74:82-89.

39. Goodnight SH Jr, Harris WS, Connor WE, Illingworth DR. Polyunsaturated fatty acids, hyperlipidemia, and thrombosis (Review). Arteriosclerosis 1982;2:87-113.

40. Sanders TAB, Sullivan DR, Reeve J, Thompson GR. Triglyceridelowering effect of marine polyunsaturates in patients with hypertriglyceridemia. Arteriosclerosis 1985;5:459-465.

41. Robins SJ, Patton GM. Separation of phospholipid molecular species by high performance liquid chromatography: potentials for use in metabolic studies (Minireview). J Lipid Res 1986;27: 131-139.
42. Robins SJ, Fasulo JM, Robins VF, Patton GM. Utilization of different fatty acids for hepatic and biliary phosphatidylcholine formation and the effect of changes in phosphatidylcholine molecular species on biliary lipid secretion. J Lipid Res 1991;32: 985-992.

43. Lorenz R, Spengler U, Fischer S, Duhm J, Weber PC. Platelet function, thromboxane formation and blood pressure control during supplementation of the Western diet with cod liver oil. Circulation 1983;67:504-511.

44. Berr F, Schreiber E, Frick U. Interrelationships of bile acid and phospholipid fatty acid species with cholesterol saturation of duodenal bile in health and gallstone disease. HEPATOLOGY 1992;16:71-81.

45. Ventura MA, Woollett LA, Spady DK. Dietary fish oil stimulates hepatic low density lipoprotein transport in the rat. J Clin Invest 1989;84:528-537.

46. Cohen DE, Angelico M, Carey MC. Structural alterations in lecithin-cholesterol vesicles following interactions with monomeric and micellar bile salts: physical-chemical basis for subselection of biliary lecithin species and aggregative states of biliary lipids during bile formation. $J$ Lipid Res 1990;31:55-70.

47. Leaf A, Weber PC. Cardiovascular effects of n-3 fatty acids (Review). N Engl J Med 1988;318:549-557.

48. Lee SP, LaMont JT, Carey MC. Role of gallbladder mucus hypersecretion in the evolution of cholesterol gallstones: studies in the prairie dog. J Clin Invest 1981;67:1712-1723.

49. O'Leary DP, LaMorte WM, Scott TE, Booker ML, Stevenson J Inhibition of prostaglandin synthesis fails to prevent gallbladder mucin hypersecretion in the cholesterol-fed prairie dog. Gastroenterology 1991;101:812-820.

50. Cohen BI, Mosbach E, Ayyad N, Yoshii M, McSherry ChK. Aspirin does not inhibit cholesterol cholelithiasis in two established animal models. Gastroenterology 1991;101:1109-1116.

51. Delchier JC, Benfredj P, Preaux AM, Metreau JM, Dhumeaux D. The usefulness of microscopic bile examination in patients with suspected microlithiasis: a prospective evaluation. HEPATOLOGY $1986 ; 6: 118-122$.

52. Ramond MJ, Dumont M, Belghiti J, Erlinger S. Sensitivity and specificity of microscopic examination of gallbladder bile for gallstone recognition and identification. Gastroenterology 1988 95:1339-1343.

53. Strasberg SM, Harvey PRC. The pathogenesis of cholesterol gallstones: vesicles and substances affecting their stability in bile. In: Paumgartner G, Stiehl A, Barbara L, Roda E, eds. Strategies for the treatment of hepatobiliary diseases. Dordrecht, The Netherlands: Kluwer Academic Publishers, 1990:109-118. 